\title{
Microbial biotechnology for bio- prospecting of microbial bioactive compounds and secondary metabolites
}

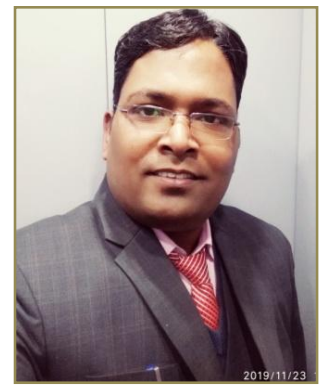

S econdary metabolites and bioactive compounds produced by beneficial soil microbiomes, plant microbiomes and extremophilic microbiomes are of great significance for agriculture, industry and humans welfare. Microbial bioactive compounds and secondary metabolites are broadly used as active ingredients in medicine, in agriculture, and in food additives [1]. Metabolism is the amount of life-sustaining chemical transformations in the cells of organisms, and metabolic intermediates are called metabolites (small molecules). Among the metabolites, the primary microbial metabolite (Ethanol, amino acids and lactic acid) is necessary for growth, reproduction and development of organisms/ producer, whereas secondary metabolites (pigments and antibiotics) produced by microorganisms are useful for other living organisms for their enhanced metabolic processes [2, 3]. The microbial secondary metabolites are useful for human welfare as these metabolites are being used in diverse biological processes in agriculture, medicines, food industry, chemical industry and related fields.

Archaea, bacteria and eukarya are three domain and microorganisms from these domain has been reported and characterized for production of potential low molecular weight natural substances and their uses in wide ranges of applications pharmaceuticals, food, agrochemical, and industries [4]. Considering the various studies of microbial bioactive compounds and secondary metabolites, it can be concluded that these microbes belongs to different phylum namely Rhodophyta, Proteobacteria, Mucoromycota, Haptophyta, Firmicutes, Eustigmatophyceae, Euryarchaeota, Dinoflagellata, Cyanobacteria, Crenarchaeota, Chlorophyta, Basidiomycota, Bacteroidetes, Bacillariophyta, Ascomycota, and Actinobacteria with most dominant genera such as Streptomyces, Aspergillus, Halococcus, Bacillus, Acremonium, Pestalotiopsis, Penicillium, Pseudomonas, Fusarium, Halobacterium,
Ajar Nath Yadav

Microbial Biotechnology Laboratory, Department of Biotechnology, Dr. Khem Singh Gill Akal College of Agriculture, Eternal University, Baru Sahib, Sirmour, Himachal Pradesh, India, Email:ajar@eternaluniversity.edu.in; ajarbiotech@gmail.com

Haloarcula and Amycolatopsis $[5,6]$. Secondary metabolites of bacteria and fungi originate from several common biosynthetic pathways, but the resulting intermediates undergo numerous enzyme-catalyzed reactions that result in products with a very diverse range of chemical structures [7]. Therefore, due to their biosynthetic origin, these secondary metabolites are appropriately classified as terpenoids, polyketides, alkaloids, non-ribosomal peptides and cytochalasines [8].

Discovery of novel and potential secondary metabolites that microbes produce has been reported worldwide from a variety of habitats, including plant-related microbiomes (rhizospheric, epiphytic, and endophytic) and microbiomes of extreme habitats (halophilic, psychrophilic/thermophilic, acidophilic/alkaliphilic, and xerophilic) belongs to diverse species of different genera such as Streptosporangium, Streptomyces, Rhodococcus, Paenibacillus, Oceanicola, Marinobacter, Fodinicurvata, Bacillus, Agrococcus, Aeromicrobium, and Actinomadura [4, 9]. Novel microbes producing potential bioactive compounds and secondary metabolites have been reported from Atlantic, compost, marine habitats, forest soil, oil wells, rhizosphere soil, phyllosphere, root tubers, salt mine, sandstone surface, sea, soil and river (Figure 1). The novel microbes produce wide range of secondary metabolites e.g. $\beta$-lactam, $\beta$-hydroxybutyrate, streptothricin, pyrrolnitrin, polyanionic, octadecylmorpholine, isoprenoid quinine, isocaryophyllene, friulimicins, erythromycin, cervinomycin, aristeromycin, aminoglycoside, alahopcin [10-12]. 


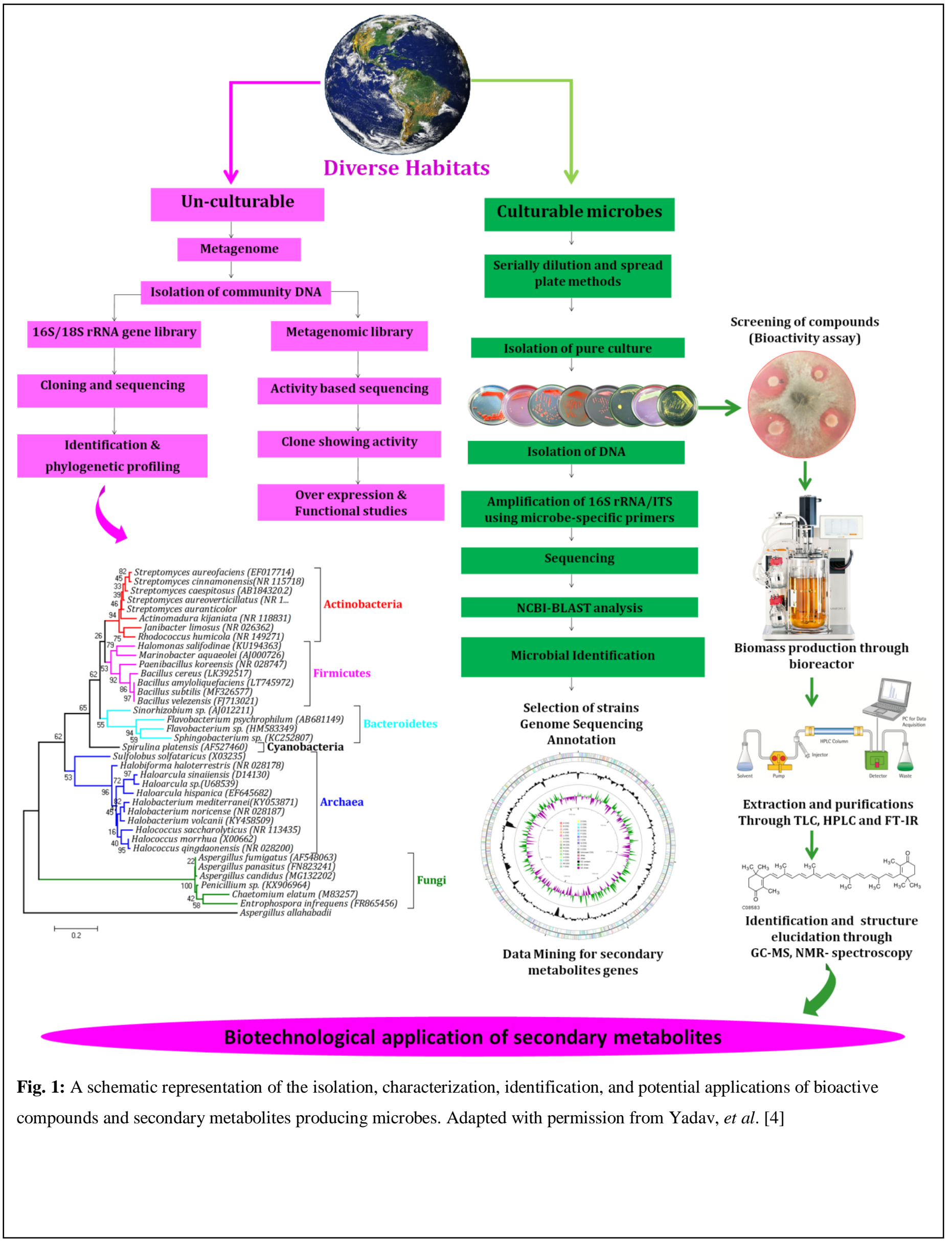




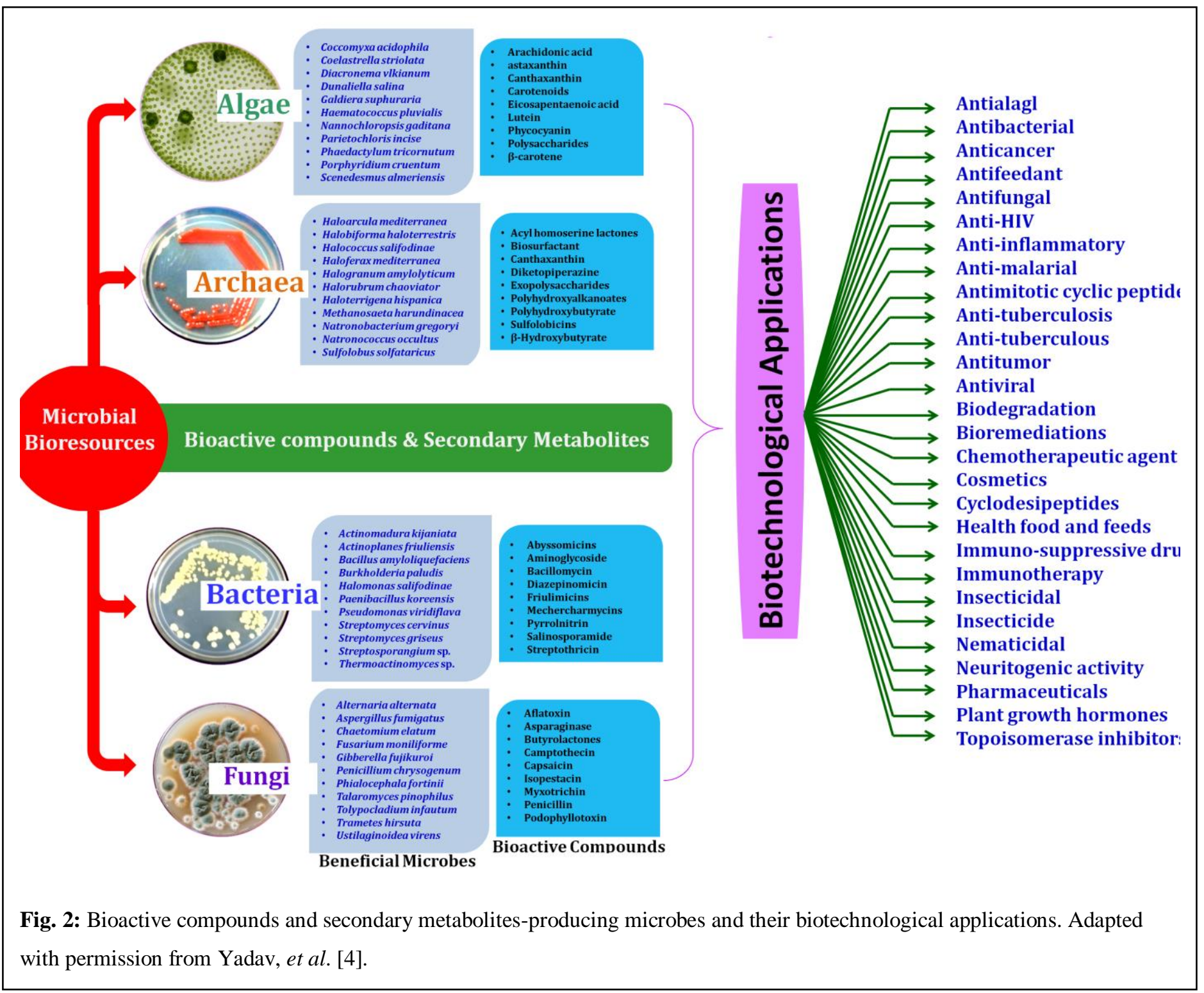

Extreme habitats represent unique ecosystems for novel and beneficial microbiomes that are producing a wide range of bioactive compounds with broadly applications in different fields [13, 14]. Microbial communities from extreme environment have ability to survive under a wide range of extreme conditions such as high/low temperatures, acidic/alkaline, saline, water deficient/stress [15]. To survive such extreme conditions, these organisms, known as extremophiles, have developed adaptive traits that allow them to grow optimally under one or more extreme conditions, while polyextremophiles grow optimally under several conditions. Polyextremophiles can grow at different abiotic stresses of water deficit, high salinity and temperature, $\mathrm{pH}$ and pressure. Natural extreme conditions can be defined as habitats that are constantly or fluctuatingly exposed to one or more other environmental factors, such as salinity, osmolarity, dehydration, UV radiation, air pressure, $\mathrm{pH}$ and temperature, microorganisms [16, 17]. Extremophilic microbiomes from many natural extreme habitats have been sorted out, and characterized, secondary metabolites production and their biotechnological applications. There are huge numbers of extremophilic microbiomes (xerophiles, psychrophiles/ thermophiles, halophiles, and acidophiles/alkaliphiles) belonging to different genera such Streptomyces, Rhodococcus, Phaeobacter, Oceanicola, Oceanibulbus, Muscodor, Micromonospora, Kitasatosporia, Fodinicurvata, Fodinicurvata, Bacillus, Amycolatopsis, Amycolatopsis, Aeromicrobium, and Actinomadura produces a wide range of secondary metabolites with potential biotechnological applications in agriculture, pharmaceuticals, chemical and food industry [18-20]. A current 
problem in the study of biological secondary metabolites/ molecules produced by extremophiles is that their potential uses may not be well known. However, this opens up huge potential for future sustainable development. The most widely researched uses by extremophiles include the production of enzymes, fatty acids and proteins, antibiotics, or biomedicine. Because survival strategies for extremophiles are often new and unique, the need to adapt microbial cellular components to extreme conditions (synthetic or natural) implies that a wide range of cellular products (metabolites and genes) are available for biotechnological applications.

Metabolomics is a key component of synthetic biology's approach for the production of microbial bioactive compounds and secondary metabolites [21]. The aim is to detect and characterize potential bioactive compounds and secondary metabolites via different metabolic pathways [22]. The fundamentals of synthetic biology are based on the concept that all living systems are composed of functional and structural modules that can be rationally designed to create potential organisms through the strain improvements [23]. However, the complexity of cells and the metabolic diversity of organisms represent a major challenge and an opportunity for the development of this interdisciplinary scientific field. Genes encoding enzymes involved in secondary metabolism are often grouped together and have a modular organization, which is an ideal target for the application of synthetic biological principles and offers almost unlimited potential for creating new cell factories, mainly through knowledge. The rules for the operation of functional blocks that control the biosynthesis of a certain metabolite are limited. Modern genetic methods have made it possible to thoroughly and quickly query the complete genome sequences of producers of bioactive compounds and secondary metabolites in order to identify loci related to the biosynthesis of metabolites. The techniques developed for genome editing and construction open the way for rational design and pathway construction and genotype modification with the primary goal of creating the desired phenotype.

Many microbial bioactive compounds and secondary metabolites are of high biotechnological value in different biological process in pharmaceuticals, medical, agricultural and food production (Figure 2). As a result of the degradation of bacterial genomes, numerous new clusters of biosynthetic genes for secondary metabolites have been discovered that encode the potential for the synthesis of a wide range of bioactive compounds and secondary metabolites that have never been observed before. Secondary metabolites are an important class of high value compounds that cover a wide range of uses, including drugs (eg antibiotics, anticancer drugs, immunosuppressants), agrochemicals (eg pesticides, insecticides, antiphedants), biofuels (eg squalene, oleoresin) and nutritional supplements (e.g. carotenoids, flavonoids, essential oils) [24]. Secondary microbial metabolites have provided a variety of pharmaceutical agents, from antibiotics to immune-suppressants. The biological synthesis of these low molecular weight compounds is not required for normal microbial growth. However, these compounds can have several benefits for the body. Fungi have the ability to produce a wide range of secondary metabolites, which usually depend on the stage of development of the fungus and environmental factors ranging from nutrient concentration to light and temperature $[25,26]$.

Agricultural productivity to meet the growing needs of the population is a major challenge for all countries [27, 28]. Using microbial bioactive compounds and secondary metabolites could achieve high crops yields and soil health for sustainable agriculture. Now-a-days there is tremendous use of aggressive biosurfactants in agricultural soils. Biosurfactants produced by microorganisms including fungi, yeasts, bacteria, and archaea could serve as green surfactants. Biosurfactants are considered less toxic and environmentally friendly. Thus, various types of biosurfactants of microbial origins could be commercially produced for a wide range of applications in the pharmaceutical, cosmetic and food industries [29]. Biosurfactants synthesized from ecological isolates also play a promising role in the agrochemical and agricultural industry. Many rhizospheric, endophytic and phyllospheric microbes produce biosurfactants [30]. These biomolecules play an important role in signaling, motility, and microbial biofilm formation, indicating that the biosurfactants controls interactions between plants and microbes. In agriculture, the potential biosurfactants can be used to eliminate plant pathogens and enhanced the bioavailability of nutrients for beneficial microbes associated with plants. Biosurfactants can be used on a large scale to improve the quality of agricultural land through soil restoration. These biomolecules could replace the harmful chemical surfactant currently used in the million dollar pesticide industry [31, 32]. Therefore, the study of biosurfactants from ecological isolates is compulsory in order to investigate their vital role in plant growth stimulation and control of phytopathogenic microorganisms. There are many conventional methods used to screen the microbial diversity and their populations for biosurfactants production. However, molecular methods encompass fewer biosurfactants from different microbial populations, and there is a need to study new biosurfactants from uncultivated microbes in the soil biosphere using advanced techniques such as functionalmetagenomics.

In conclusion, in recent years, microbial bioactive compounds and microbial secondary metabolites have been in high demand for agriculture, pharmaceutical and food industries for sustainable development. Microorganisms synthesize bioactive compounds and industrially important 
hydrolytic enzymes for different biological processes. Bioactive compounds and microbial secondary metabolites are very important for human health which includes anticancer drugs, cholesterol-lowering drugs, antibiotics, and others. The field of applications of microbial bioactive compounds and secondary metabolites for agricultural and biotechnological applications has expanded now-a-days. A huge number of such molecules have been reported from diverse groups of microorganisms; however the new discovery could be possible due to diverse microbial biotechnologies and strain improvements through recombinant DNA technologies to accelerate the pace of discovery and development.

\section{ACKNOWLEDGEMENTS}

The authors are grateful to the Department of Biotechnology, Dr. Khem Singh Gill Akal College of Agriculture, Eternal University, Baru Sahib and Department of Environment, Science and Technology (DEST), Shimla funded project "Development of microbial consortium as bioinoculants for drought and low temperature growing crops for organic farming in Himachal Pradesh" for providing the facilities and financial support, to undertake the investigations.

\section{CONFLICTS OF INTEREST}

There are no conflicts of interest.

\section{REFERENCES}

1. Berdy J. Bioactive microbial metabolites. J Antibiot. 2005; 58(1):1-26.

2. Demain AL. Microbial production of primary metabolites. Naturwissenschaften. 1980; 67(12):582-587.

3. Desire MH, Bernard F, Forsah MR, Assang CT, Denis ON. Enzymes and qualitative phytochemical screening of endophytic fungi isolated from Lantana camara Linn. Leaves. J App Biol Biotech. 2014; 2: 1-6.

4. Yadav AN, Kour D, Rana KL, Yadav N, Singh B, Chauhan VS, Rastegari AA, Hesham AE-L, Gupta VK: Metabolic Engineering to Synthetic Biology of Secondary Metabolites Production. In: New and Future Developments in Microbial Biotechnology and Bioengineering. Edited by Gupta VK, Pandey A. Amsterdam: Elsevier; 2019: 279-320.

5. Ruiz B, Chávez A, Forero A, García-Huante Y, Romero A, Sánchez M, Rocha D, Sánchez B, Rodríguez-Sanoja R, Sánchez S. Production of microbial secondary metabolites: regulation by the carbon source. Crit Rev Microbiol. 2010; 36(2):146-167.

6. Barrios-Gonzalez J, Fernandez F, Tomasini A. Microbial secondary metabolites production and strain improvement. Indian J Biotechnol. 2003; 2(3):322-333.

7. Gunatilaka A, Wijeratne E. Natural products from bacteria and fungi. Encyclopedia of life science systems (EOLSS) Eolss Publishers Co, UK. 2000.

8. Rana KL, Kour D, Yadav AN. Endophytic microbiomes: biodiversity, ecological significance and biotechnological applications. Res J Biotechnol. 2019; 14:142-162.

9. Rastegari AA, Yadav AN, Yadav N, Tataei Sarshari N: Bioengineering of Secondary Metabolites. In: New and Future Developments in Microbial Biotechnology and Bioengineering. Edited by Gupta VK, Pandey A. Amsterdam: Elsevier; 2019: 55-68.

10. Yadav AN, Verma P, Kumar V, Sangwan P, Mishra S, Panjiar N, Gupta VK, Saxena AK: Biodiversity of the Genus Penicillium in Different Habitats. In: New and Future Developments in Microbial Biotechnology and Bioengineering, Penicillium System Properties and
Applications. Edited by Gupta VK, Rodriguez-Couto S. Amsterdam: Elsevier; 2018: 3-18.

11. Firáková S, Šturdíková M, Múčková M. Bioactive secondary metabolites produced by microorganisms associated with plants. Biologia. 2007; 62(3):251-257.

12. Kour D, Rana KL, Kaur T, Singh B, Chauhan VS, Kumar A, Rastegari AA, Yadav N, Yadav AN, Gupta VK: Extremophiles for Hydrolytic Enzymes Productions: Biodiversity and Potential Biotechnological Applications. In: Bioprocessing for Biomolecules Production. Edited by Molina G, Gupta VK, Singh B, Gathergood N; 2019: 321-372.

13. Kour D, Kaur T, Devi R, Rana KL, Yadav N, Rastegari AA, Yadav AN: Biotechnological applications of beneficial microbiomes for evergreen agriculture and human health. In: Trends of Microbial Biotechnology for Sustainable Agriculture and Biomedicine Systems: Perspectives for Human Health. Edited by Rastegari AA, Yadav AN, Yadav N. Amsterdam: Elsevier; 2020: 255-279.

14. Yadav AN, Kour D, Kaur T, Devi R, Guleria G, Rana KL, Yadav N, Rastegari AA: Microbial biotechnology for sustainable biomedicine systems: Current research and future challenges. In: Trends of Microbial Biotechnology for Sustainable Agriculture and Biomedicine Systems: Perspectives for Human Health. Edited by Rastegari AA, Yadav AN, Yadav N. Amsterdam: Elsevier; 2020: 281-292.

15. Yadav AN, Saxena AK. Biodiversity and biotechnological applications of halophilic microbes for sustainable agriculture. J Appl Biol Biotechnol. 2018; 6(1):48-55.

16. Petersen L-E, Kellermann MY, Schupp PJ: Secondary Metabolites of Marine Microbes: From Natural Products Chemistry to Chemical Ecology. In: YOUMARES 9 - The Oceans: Our Research, Our Future: Proceedings of the 2018 conference for YOUng MArine RESearcher in Oldenburg, Germany. Edited by Jungblut S, Liebich V, Bode-Dalby M. Cham: Springer International Publishing; 2020: 159-180.

17. Yadav AN, Kumar R, Kumar S, Kumar V, Sugitha T, Singh B, Chauhan V, Dhaliwal HS, Saxena AK. Beneficial microbiomes: biodiversity and potential biotechnological applications for sustainable agriculture and human health. J Appl Biol Biotechnol. 2017; 5(6):4557.

18. Chaabouni I, Guesmi A, Cherif A: Secondary Metabolites of Bacillus: Potentials in Biotechnology. In: Bacillus thuringiensis Biotechnology. Edited by Sansinenea E. Dordrecht: Springer Netherlands; 2012: $347-$ 366.

19. Yadav AN, Yadav N, Sachan SG, Saxena AK. Biodiversity of psychrotrophic microbes and their biotechnological applications. J Appl Biol Biotechnol. 2019; 7(04):99-108.

20. Vemireddy B, Madasi A, Ajmeera A, Vanteru KR. Distribution and diversity of endophytic fungi associated with three medicinal tree species from Eturnagaram Wildlife Sanctuary, TS, India. J App Biol Biotech. 2020; 8(6):7-12.

21. Dwivedi GR, Sisodia BS, Shikha: Secondary Metabolites: Metabolomics for Secondary Metabolites. In: New and Future Developments in Microbial Biotechnology and Bioengineering. Edited by Gupta VK, Pandey A. Amsterdam: Elsevier; 2019: 333-344.

22. Jones JA, Toparlak ÖD, Koffas MA. Metabolic pathway balancing and its role in the production of biofuels and chemicals. Curr Opin Biotechnol. 2015; 33:52-59.

23. Rastegari AA, Yadav AN, Yadav N: Genetic Manipulation of Secondary Metabolites Producers. In: New and Future Developments in Microbial Biotechnology and Bioengineering. Edited by Gupta VK, Pandey A. Amsterdam: Elsevier; 2019: 13-29.

24. Giddings L-A, Newman DJ. Microbial natural products: molecular blueprints for antitumor drugs. J Ind Microbiol Biotechnol. 2013; 40(11):1181-1210.

25. Devi R, Kaur T, Guleria G, Rana K, Kour D, Yadav N, Yadav A, Saxena A: Fungal secondary metabolites and their biotechnological application for human health. In: Trends of Microbial Biotechnology for Sustainable Agriculture and Biomedicine Systems: Perspectives for Human Health. Edited by Rastegari AA, Yadav AN, Yadav N. Amsterdam: Elsevier; 2020: 147-161. 
26. Amoozegar MA, Safarpour A, Noghabi KA, Bakhtiary T, Ventosa A. Halophiles and Their Vast Potential in Biofuel Production. Front Microbiol. 2019; 10(1895).

27. Kour D, Rana KL, Kaur T, Yadav N, Yadav AN, Kumar M, Kumar V, Dhaliwal HS, Saxena AK. Biodiversity, current developments and potential biotechnological applications of phosphorus-solubilizing and -mobilizing microbes: A review. Pedosphere. 2021; 31(1):43-75.

28. Kour D, Rana KL, Yadav AN, Yadav N, Kumar M, Kumar V, Vyas P, Dhaliwal HS, Saxena AK. Microbial biofertilizers: Bioresources and eco-friendly technologies for agricultural and environmental sustainability. Biocatal Agric Biotechnol. 2020; 23:101487.

29. Sachdev DP, Cameotra SS. Biosurfactants in agriculture. Appl Microbiol Biotechnol. 2013; 97(3):1005-1016.

30. Yadav AN. Plant microbiomes for sustainable agriculture: current research and future challenges. In: Plant Microbiomes for Sustainable Agriculture. Edited by Yadav AN, Singh J, Rastegari AA, Yadav N. Cham: Springer International Publishing; 2020: 475-482.

31. Thakur N, Kaur S, Tomar P, Thakur S, Yadav AN: Microbial biopesticides: Current status and advancement for sustainable agriculture and environment. In: Trends of Microbial Biotechnology for Sustainable Agriculture and Biomedicine Systems: Diversity and Functional Perspectives. Edited by Rastegari AA, Yadav AN, Yadav N. Amsterdam: Elsevier; 2020: 243-282.
32. Kumar M, Yadav AN, Saxena R, Paul D, Tomar RS. Biodiversity of pesticides degrading microbial communities and their environmental impact. Biocatal Agri Biotech. 2021; 31:101883.

\section{How to cite this article:}

Yadav AN. Microbial biotechnology for bio-prospecting of microbial bioactive compounds and secondary metabolites. J App Biol Biotech. 2021; 9 (2): 1-6.

DOI: $10.7324 / J A B B .2021 .92 \mathrm{ed}$ 